\title{
Methyl Helicterate Inhibits Hepatic Stellate Cell Activation Through Modulation of Apoptosis and Autophagy
}

\author{
Xiao-Lin Zhang ${ }^{\mathrm{a}}$ Zhao-Ni Chen ${ }^{\mathrm{a}} \quad$ Quan-Fang Huang $^{\mathrm{b}} \quad$ Fa-Cheng Bai ${ }^{\mathrm{a}}$ \\ Jin-Lan Nie ${ }^{a}$ Sheng-Juan Lu ${ }^{a}$ Jin-Bin Wei ${ }^{a}$ Xing Lin ${ }^{a}$

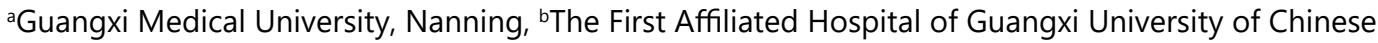 \\ Medicine, Nanning, China
}

\section{Key Words}

Methyl helicterate $\cdot$ Hepatic stellate cells $•$ Apoptosis • Autophagy

\begin{abstract}
Background/Aims: Activated hepatic stellate cells (HSCs) are the major source of extracellular matrix (ECM). Therefore inhibiting HSC activation is considered as an effective strategy to inhibit the process of liver fibrosis. This study aimed to investigate the underlying mechanism of methyl helicterate $(\mathrm{MH})$ isolated from Helicteres angustifolia on the activation of HSCs. Methods: HSC-T6 cells were treated with various concentration of $\mathrm{MH}$ and autophagy was inhibited by 3-Methyl adenine (3-MA) or RNA interference. Cell viability was observed by MTT assay and cell colony assay. Cell cycle and apoptosis were analyzed using flow cytometry. Autophagic vacuoles were observed by transmission electron microscopy and monodansyl cadaverine (MDC) staining. Moreover, autophagy-related genes and proteins were detected using real-time PCR and Western blot assays, respectively. Results: $\mathrm{MH}$ significantly inhibited HSC activation, as evidenced by the inhibition of cell viability, colony formation and the expression of $\alpha$-SMA and collagen I. MH caused cell cycle arrest in G2/M phase. Moreover, $\mathrm{MH}$ significantly induced apoptosis through regulating the mitochondria-dependent pathway and the activity of caspases. $\mathrm{MH}$ treatment significantly increased lysosomes and autophagosomes, and enhanced the formation of autophagic vacuoles and autophagic flux. Interestingly, inhibiting autophagy by 3-MA or RNA interference abolished the ability of MH in inhibiting HSC activation. On the other hand, induction of autophagy promoted $\mathrm{MH}$-induced HSC apoptosis. Further study showed that $\mathrm{MH}$-induced HSC apoptosis and autophagy was mediated by the JNK and PI3K/Akt/mTOR pathways. Conclusion: Our results demonstrate that $\mathrm{MH}$-induced HSC apoptosis and autophagy may be one of the important mechanisms for its anti-fibrosis effect.




\section{Cellular Physiology Cell Physiol Biochem 2018;51:897-908 \begin{tabular}{ll|l} 
and Biochemistry & $\begin{array}{l}\text { DOI: } 10.1159 / 000495390 \\
\text { Published online: } 22 \text { November } 2018\end{array}$ & $\begin{array}{l}\text { (c) } 2018 \text { The Author(s). Published by S. Karger AG, Basel } \\
\text { www.karger.com/cpb }\end{array}$
\end{tabular} \\ Zhang et al.: Methyl Helicterate Inhibits HSC Activation}

\section{Introduction}

Hepatic fibrosis characterized by excessive accumulation of extracellular matrix (ECM) is an essential pathophysiologic consequence of chronic hepatic injury [1]. Hepatic stellate cells (HSCs) are the main source of ECM. Upon chronic liver injury, HSCs transdifferentiate from quiescent, lipid droplet-containing cells toward myofibroblast-like cells with an increased proliferation rate, decreased number of lipid droplets and high production of ECM. HSC activation is a critical event in hepatic fibrogenesis, and therefore suppressing HSC activation has been considered an important strategy to intervene the process of liver fibrosis [2].

Recent studies demonstrated that autophagy may play a double-role in hepatic diseases including liver fibrosis. On one hand, autophagy acted as a player that induced HSC activation and exacerbated liver fibrosis [3], which supplied energy for activation of HSCs by delivering triglycerides and other components. Therefore, many researchers believe that suppression of autophagy may be a promising therapeutic target for the treatment of liver fibrosis [4]. But, on the other hand, it was already shown that inhibition of autophagy (through deleting the autophagy-related genes or using autophagy inhibitors) reduced the activation of HSC, which led to reduced fibrosis [5]. These contradictory results of the previous studies suggest that autophagy is a double-edged sword: appropriate autophagy level is considered as a selfprotection mechanism, whereas excessive autophagy induction can lead to cell death [6].

Natural products recently have attracted extensive attention in the prevention and treatment of liver fibrosis. Helicteres angustifolia (Sterculiaceae) has been widely used in traditional Chinese medicine for the treatment of immune disorders and liver diseases. We previously have isolated an active ingredient from this herb and identified it as methyl helicterate $(\mathrm{MH})$. We found that $\mathrm{MH}$ significantly alleviated $\mathrm{CCl}_{4}$-induced hepatic injury in rats by suppressing oxidative stress, NF- $\kappa \mathrm{B}$ activation and Fas/FasL pathway [7], and it also notably ameliorated liver injury induced by hepatitis B virus [8]. Moreover, MH significantly ameliorated $\mathrm{CCl}_{4}$-induced hepatic fibrosis in rats through modulation of TGF- $\beta / \mathrm{Smad} 3$ signaling pathway [9]. However, its protective mechanism on hepatic fibrosis, especially on hepatic stellate cells, remains unclear so far. In our recent pilot experiment, we found that MH markedly induced HSC-T6 cell apoptosis and autophagic cell death, suggesting that the protective effect of $\mathrm{MH}$ on hepatic fibrosis may be associated with the regulation of apoptosis and autophagy.

In the present study, to verify our hypothesis, we should analyze the processes of autophagy and apoptosis triggered by MH and the role of the JNK and PI3K/Akt/mTOR signaling pathways in these two events in HSCs.

\section{Materials and Methods}

Cytotoxicity test

MTT assay was performed to assess the cytotoxicity of methyl helicterate (MH) on HSC-T6 cells. Briefly, HSC-T6 cells were seeded in 96-well plates $\left(1 \times 10^{6}\right.$ cells/well) for $24 \mathrm{~h}$, and then were treated with various concentration of MH for $24 \mathrm{~h}$; finally, $100 \mu \mathrm{L}$ MTT ( $5 \mathrm{mg} / \mathrm{mL}$ ) was added to each well and incubated for 4 $\mathrm{h}$. The absorbance was measured at $570 \mathrm{~nm}$ using a microplate reader (Bio-Tek, USA). The half maximal inhibitory concentration $\left(\mathrm{IC}_{50}\right)$ values were calculated.

\section{Cell culture and treatment}

HSC-T6 cells were cultured in Dulbecco's modified eagle medium (DMEM) [10]. Cells were treated with equal amount of culture medium (normal control) or $\mathrm{MH}(3,6$, and $12 \mu \mathrm{mol} / \mathrm{l}$ ) in the absence or presence of various agents, including E64d (10 $\mathrm{g} / \mathrm{ml}$, Cayman Chemical), Pep A (10 $\mu \mathrm{g} / \mathrm{ml}$, Sigma), 3-Methyl adenine (3-MA; 5 mmol/l, Sigma), Rapamycin (Rap; 10 nmol/l, Sigma), LY294002 (25 mol/l, ALEXIS Biochemicals), and SP600125 (10 $\mu \mathrm{mol} / \mathrm{l}$, Cayman Chemical). 


\section{\begin{tabular}{ll} 
Cellular Physiology & Cell Physiol Biochem 2018;51:897-908 \\
\hline Dol: 10.1159/000495390 & 2018 The Author(s). Published by S. Karger AG, Basel
\end{tabular}

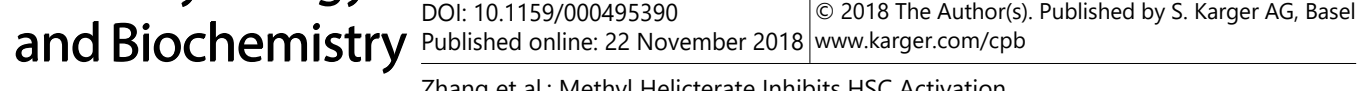

Cell colony assay

The effect of MH on cell colony was observed as previously described [11]. In brief, HSC-T6 cells were seeded into 6 -well plates $\left(1 \times 10^{3}\right.$ cells/well $)$ and treated with various concentration of MH. Following a further 5 days of incubation, cells were fixed with methanol and stained with crystal violet. Colony forming unites that compose of fifty or more than were counted.

Cell cycle analysis

Cell cycle was analyzed using flow cytometry as described previously [12]. Briefly, HSC-T6 cells were treated with $\mathrm{MH}$ for $24 \mathrm{~h}$. Then cells were suspended, washed with PBS, centrifuged, and fixed in $70 \%$ ethanol (v/v) at $-20^{\circ} \mathrm{C}$ overnight. Finally, the cells were stained with propidium iodide (PI) solution $(20 \mathrm{mg} /$ $\mathrm{mL} \mathrm{PI} \mathrm{and} 250 \mathrm{mg} / \mathrm{mL}$ RNAse in a solution of $0.1 \%$ Triton X-100 in PBS) for $1 \mathrm{~h}$ at $50^{\circ} \mathrm{C}$. Cell cycle analysis of PI-stained cells was performed using a FACScan flow cytometer (Becton Dickinson).

\section{Apoptosis analysis}

HSC-T6 cells were seeded in 6-well plates $\left(3 \times 10^{5}\right.$ cells/well) overnight, then treated with MH as described above for $24 \mathrm{~h}$. Annexin V-FITC $(10 \mu \mathrm{l})$ and PI solution $(10 \mu \mathrm{l})$ were added and incubated for $20 \mathrm{~min}$ at room temperature in dark. A total of $400 \mu \mathrm{l}$ binding buffer was then added, and the cells were immediately analyzed with a flow cytometer (Becton Dickinson) [12].

Detection of mitochondrial membrane potential (MMP)

MMP was detected as previously described [13]. Briefly, HSC-T6 cells were seeded in 12 well-plates $\left(3 \times 10^{5}\right.$ cells/well) overnight and treated with MH (3, 6, and $\left.12 \mu \mathrm{M}\right)$ for $24 \mathrm{~h}$. About $500 \mu \mathrm{l}$ of JC-1 $(5,5,6$, 6'-tetrachloro -1, 1',3, 3'- tetraethylbenzimi- dazolylcarbocyanine iodide) was added into each well for 20 min and MMP was then measured using a FACScan flow cytometry (Becton Dickson) [14].

\section{Transmission electron microscopy}

Cells were serum-starved for $12 \mathrm{~h}$ and treated with $\mathrm{MH}$ as described above for $24 \mathrm{~h}$ for morphological examination using transmission electron microscopy, as previously described [10].

\section{Detection of autophagic vacuoles}

Cells were serum-starved for $12 \mathrm{~h}$ and treated with $\mathrm{MH}$ as described above for $24 \mathrm{~h}$. Then, cells were incubated with $0.05 \mathrm{mM}$ monodansyl cadaverine (MDC) in PBS for $10 \mathrm{~min}$ at $37^{\circ} \mathrm{C}$. The cells were next washed 4 times with PBS and observed under a fluorescence microscope at excitation wavelength of 380 $\mathrm{nm}$ and emission wavelength of $525 \mathrm{~nm}$ as previously described [10].

\section{RNA interference}

siRNAs against human Atg5 (sense: 5'-GAC GUU GGU AAC UGA CAA ATT-3', and antisense: 5'-UUU GUC AGU UAC CAA CGU CTT-3') were purchased from Genepharma (Shanghai, China). The control siRNA or Atg5-siRNA was transfected with Lipofectamine 2000 reagent (Invitrogen Inc., California, USA) for $24 \mathrm{~h}$ in HSC-T6 cells according to the manufacturer's protocol. Forty-eight hours later, the medium was renewed and the cells were rinsed with PBS thrice and extracted for sodium dodecyl sulphate polyacrylamide gel electrophoresis (SDS-PAGE) in lysis buffer.

Real-time reverse transcription polymerase chain reaction (RT-PCR)

Total RNA was extracted from cells by TRIzol reagent (Life Technologies, Inc.) and RT-PCR assay was performed as described previously [15]. The primers were as follows: Atg5: $5^{\prime}$-GGC CAT CAA TCG GAA ACT CAT-3' and 5'-CAG CCA CAG GAC GAA ACA GC- $3^{\prime}$; Atg7: $5^{\prime}$-GGC TCA TCG CTT TTT GCC A AC ATC-3' and $5^{\prime}$ GCA CTG CTG GTC CAA GGT CCG- $3^{\prime}$; $\beta$-actin: $5^{\prime}$-ACT TA G TTG CGT TAC ACC CTT-3' and $5^{\prime}$-GTC ACC TTC ACC GTT CCA-3'. 
Western blot

Western blot assay was performed as described previously [12]. Briefly, the total proteins were isolated from cells using RIPA buffer (Thermo Fischer Scientific, Inc., Waltham, MA) according to the manufacturer's instruction. The protein was separated on $10 \%$ sodium dodecyl sulfate (SDS)-polyacrylamide gels, and then transferred onto nitrocellulose membranes (Millipore Corporation, USA). The membrane was incubated with the primary antibodies for $24 \mathrm{~h}$ at $4{ }^{\circ} \mathrm{C}$, including $\alpha$-SMA (1:400; Bioss, China), collagen-I (1:1000; Abcam, USA), cleaved PAPR (1:500), cleaved caspase-3 (1:500), cleaved caspase-8 (1:500), Atg5 (1:500), Atg7 (1:500), Beclin-1 (1:500), LC3 I/II (1:500), P62 (1:500) (Cell Signaling Technology), Bcl-2 (1:500), Bax (1:500), cytochrome c (1:500) (Santa Cruz Biotechnology), PI3K (1:400), p-PI3K (1:400), p-Akt (1:400), Akt (1:400), p-mTOR (1:400), mTOR (1:400), p-p70S6k (1:400), p70S6k (1:400) (Bioworld Technology Inc.), p-JNK (1:400), JNK (1:400), p-c-Jun (1:400), c-Jun (1:400) (Epitomic, San Francisco, California, USA), and GAPDH (1:500; Santa Cruz Biotechnology), and then incubated with the secondary antibodies conjugated to horseradish peroxidase (HRP) for $30 \mathrm{~min}$ at room temperature. Following washing and preincubation in staining buffer, the colorimetric reaction was developed by incubating the membranes with HRP substrate. The bands were scanned and quantified by NIH IMAGEJ 1.38 Software (US National Institutes of Health, Bethesda). The detected proteins were normalized to GAPDH or the respective total protein, as appropriate.

\section{Statistical analysis}

Statistical analysis was carried out using the Software of SPSS (Ver.11.5) as described in our previous study [16]. Differences between the groups were assessed using a one-way analysis of variance (ANOVA) with a Tukey's test for post hoc multiple comparisons. The data are presented as the means \pm SD. A $p$-value $<0.05$ was considered statistically significant.

\section{Results}

\section{MH inhibited HSC activation}

The result showed that MH significantly reduced cell viability in a concentrationdependent manner and its $\mathrm{IC}_{50}$ was $13.57 \pm 3.63 \mu \mathrm{M}$ (Fig. $1 \mathrm{~A}$ ). Therefore, the concentration 3 , 6 and $12 \mu \mathrm{M}$ were chose for the following experiments.

Activated HSCs play a pivotal role in liver fibrosis. We observed that $\mathrm{MH}$ inhibited the colony formation of HSC-T6 cells in a concentration-dependent manner (Fig. 1B). Moreover, we determined the expression of $\alpha$-SMA and collagen I to assess the activation status of HSCs and found that $\mathrm{MH}$ significantly reduced the expression of $\alpha$-SMA and collagen I in HSC-T6 cells (Fig. 1C and D), suggesting that $\mathrm{MH}$ can inhibit HSC activation.

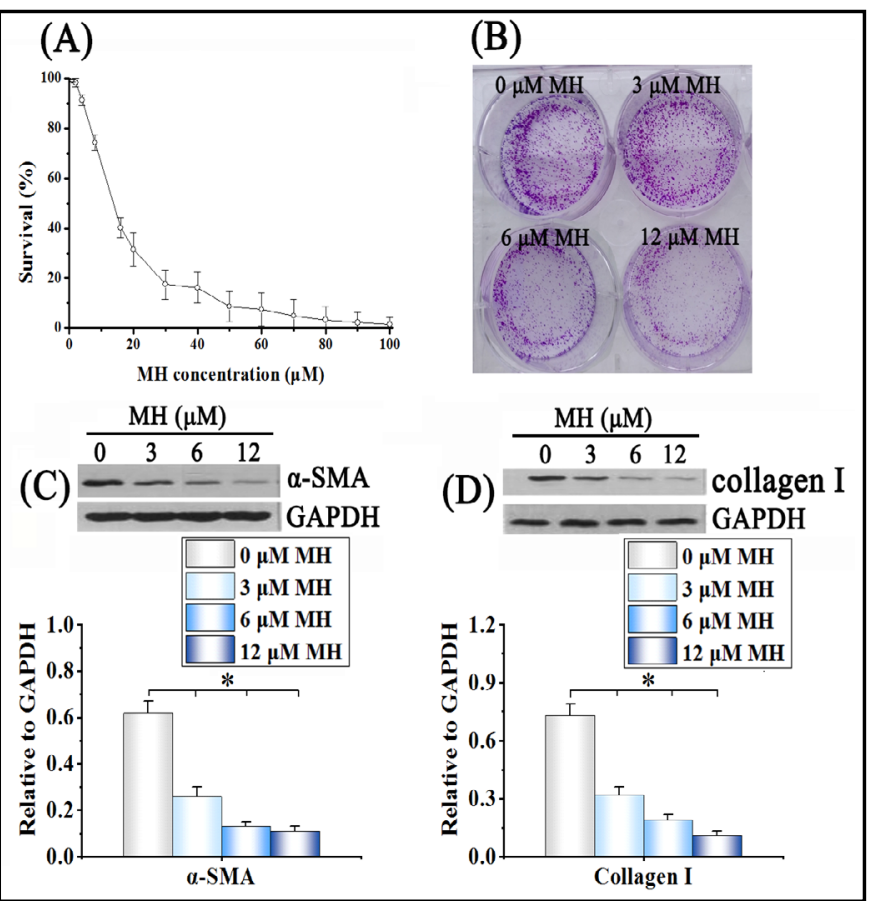

Fig. 1. MH significantly inhibited HSC-T6 cell activation. (A) Cell vitality was detected by MTT assay. (B) The colony formation of HSC-T6 cell was observed using clonogenecity assay. (C) - (D) The expression of $\alpha$-SMA and collagen I in HSC-T6 cells were detected by Western blotting. ${ }^{*} \mathrm{P}<0.05$ vs. the untreated control $(0 \mu \mathrm{M} \mathrm{MH})$. 


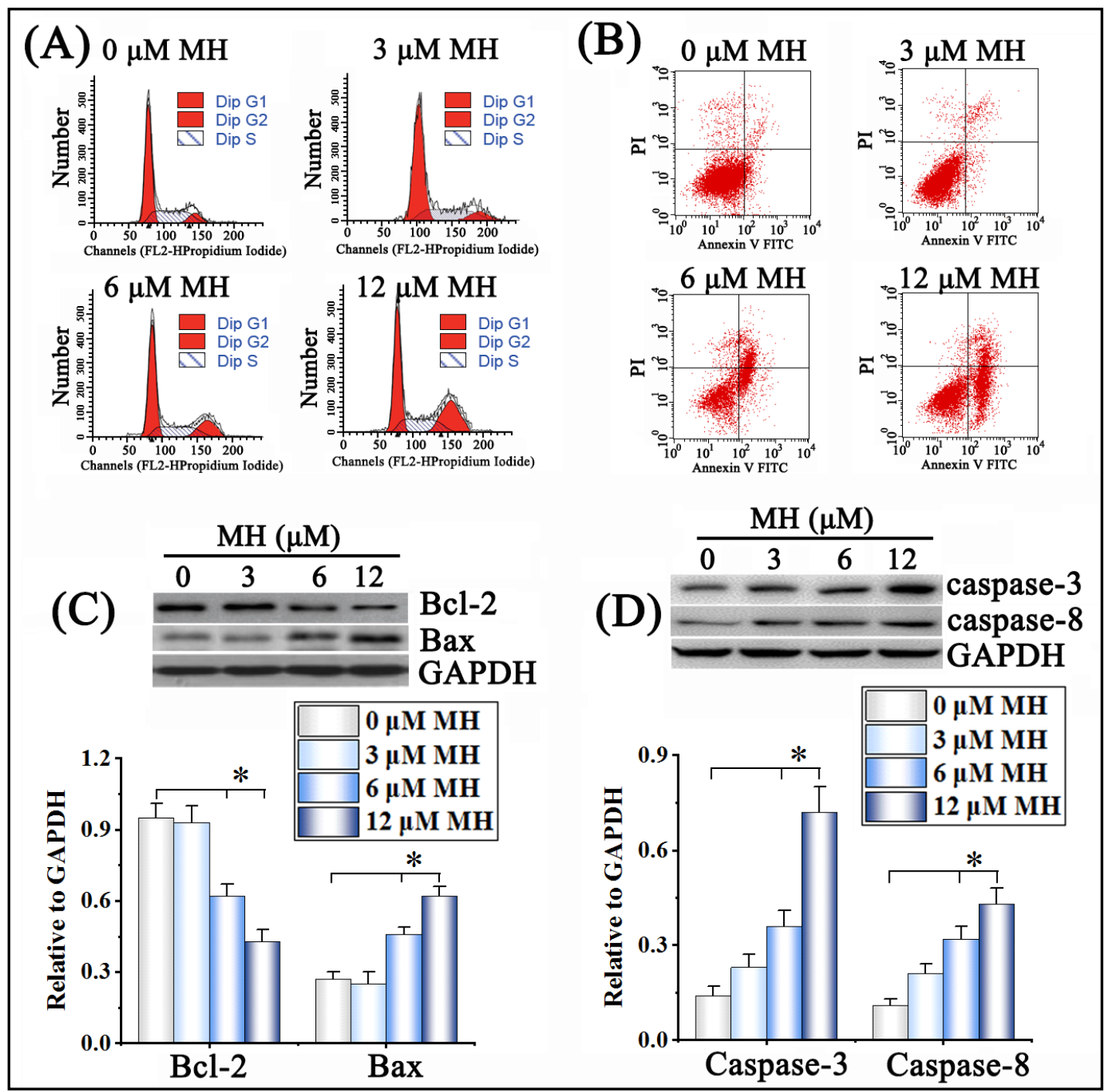

Fig. 2. MH significantly induced cell cycle arrest at G2/M phase and promoted apoptosis in HSC-T6 cells. (A) Cell cycle was detected by flow cytometry. (B) Cell apoptosis was detected by flow cytometry. (C) The expression of Bcl-2 and Bax were detected by Western blotting. (D) The expression of caspase- 3 and -8 were detected by Western blotting. ${ }^{*} \mathrm{P}<0.05$ vs. the untreated control $(0 \mu \mathrm{M} \mathrm{MH})$.

\section{MH induced cell cycle arrest at G2/M phase}

The cell cycle distribution was detected by flow cytometry. The result showed that treatment with MH led to an obvious G2/M arrest, suggesting that MH exerts its antiproliferative effect maybe by inhibiting the cell cycle (Fig. 2A).

\section{MH induced HSCs apoptosis}

The flow cytometry analysis showed that MH significantly promoted cell apoptosis in a concentration-dependent manner (Fig. 2B). Furthermore, MH significantly decreased Bcl-2 expression, whereas increased Bax expression (Fig. 2C). MH markedly increased the expression of caspase-3 and -8 in HSC-T6 cells (Fig. 2D). These results suggested that MH induces HSC apoptosis likely by modulating the expression of apoptosis-related proteins and the activation of caspase-3 and -8. 


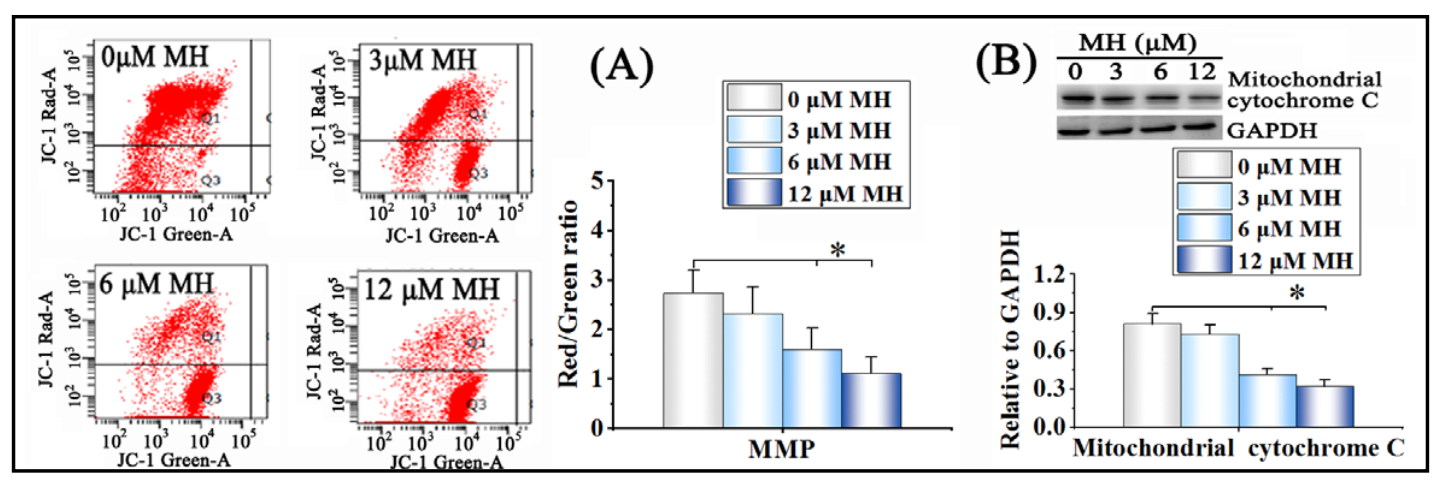

Fig. 3. MH induced mitochondrial dysfunction. (A) Mitochondrial membrane potential (MMP) was measured by JC-1 staining. (B) Mitochondrial cytochrome $\mathrm{C}$ was detected using Western blotting. ${ }^{*} \mathrm{P}<0.05$ vs. the untreated control $(0 \mu \mathrm{M} \mathrm{MH})$.

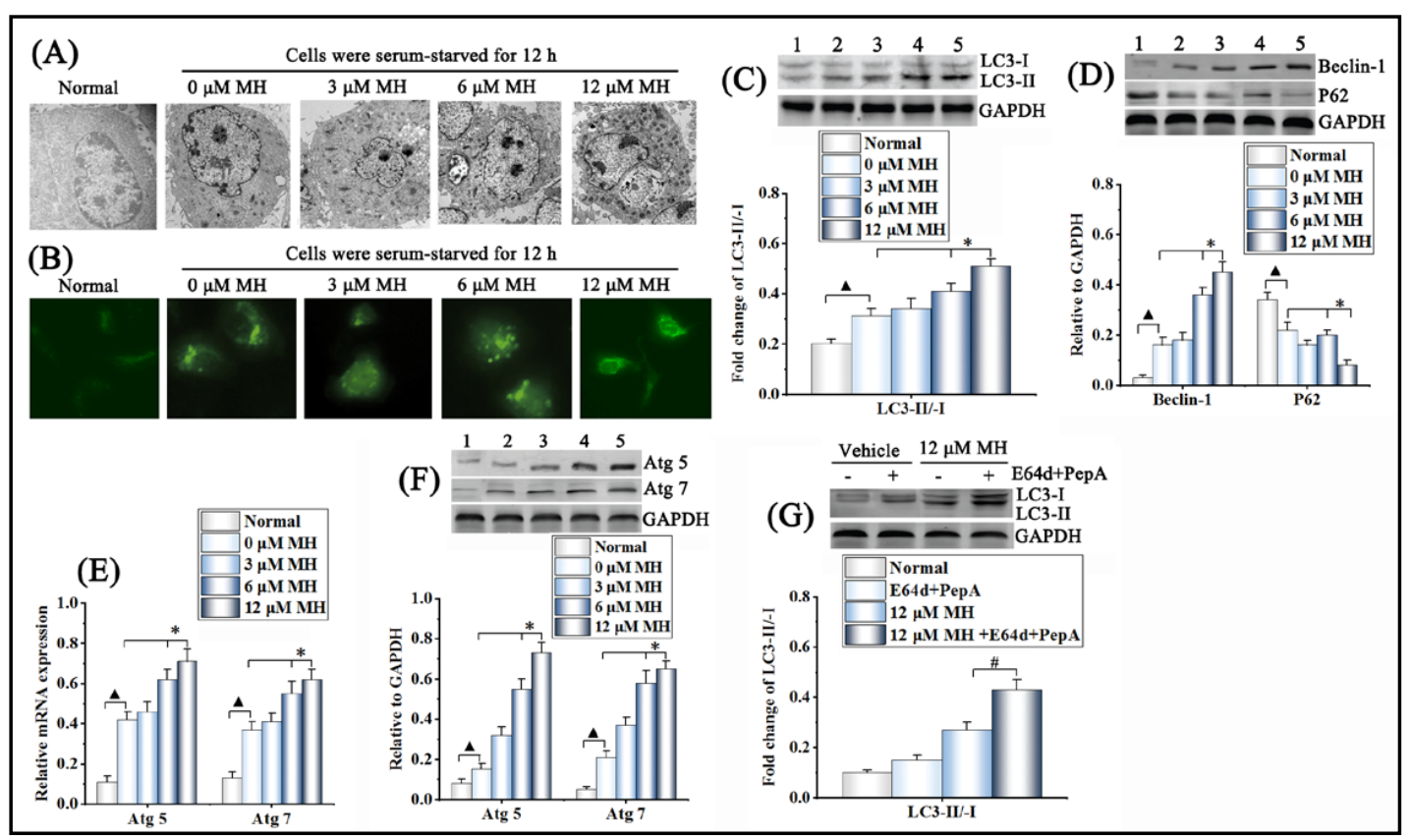

Fig. 4. MH significantly induced HSC autophagy. (A) Autophagosomes were observed using electron microscopy. (B) The formation of autophagic vacuoles was observed using an autofluorescent monodansyl cadaverine dye. (C) - (F) MH significantly increased the level of the autophagic indicators; band 1 to 5 represented the normal control, and $0,3,6$ and $12 \mu \mathrm{M} \mathrm{MH}$, respectively; ${ }^{\Delta} \mathrm{P}<0.05$ vs. the normal control and ${ }^{*} \mathrm{P}<0.05$ vs. untreated control $(0 \mu \mathrm{M} \mathrm{MH})$. (G) $\mathrm{MH}$ markedly enhanced autophagic flux; ${ }^{\mathrm{P}}<0.05$ vs. $12 \mu \mathrm{M}$ MH alone.

\section{MH caused mitochondrial dysfunction}

Mitochondrial dysfunction is one of the mechanisms for induction of apoptosis. In the present study, we found that MH decreased the mitochondrial membrane potential (MMP) in a dose-dependent manner (Fig. 3A). Meanwhile, MH significantly promoted the release of cytochrome $\mathrm{C}$ from mitochondria to cytoplasm, resulting in a decrease in mitochondrial cytochrome C level (Fig. 3B). These results suggested that MH promotes HSC apoptosis partially through destroying the mitochondrial function. 
MH induced HSC autophagy

Excessive autophagy may induce autophagic cell death. In the present study, we firstly observed autophagosomes using electron microscopy and found that MH treatment significantly increased lysosomes and autophagosomes (Fig. 4A). Moreover, by using the autofluorescent monodansyl cadaverine dye, we found that MH significantly increased the formation of autophagic vacuoles in HSC-T6 cells (Fig. 4B). Furthermore, we analyzed the important biomarkers of autophagosome formation. We found that $\mathrm{MH}$ notably increased the conversion of LC3-I to LC3-II, upregulated the protein expressions of Beclin-1, and decreased the expression of P62 (Fig. 4C and D); furthermore, MH significantly enhanced the mRNA and protein expressions of Atg 5 and 7 (Fig. 4E and F). In addition, we further assessed the effect of $\mathrm{MH}$ on autophagic flux. E64d and Pep A are the lysosomal protease inhibitors that interfere with the fusion between autophagosomes and lysosomes [17]. In our experiment, HSC-T6 cells were pretreated with E64d + Pep A for $1 \mathrm{~h}$ and then were treated with $\mathrm{MH}$ for $24 \mathrm{~h}$. We found that the two inhibitors induced a further increase in the MH-induced accumulation of LC3-II (Fig. 4G), suggesting that MH can enhance autophagic flux. Taken together, our findings indicated that MH induces an autophagic response in HSCs.

Fig. 5. Inhibition of autophagy decreased the ability of $\mathrm{MH}$ in inhibiting HSC activation. (A) HSC-T6 cells were pretreated with 3-MA for $12 \mathrm{~h}$ and then incubated with $\mathrm{MH}$ for 24 h. (B) Cells were transfected with Atg5-siRNA for 24 $\mathrm{h}$ and then treated with $\mathrm{MH}$ for $24 \mathrm{~h}$. ${ }^{\#} \mathrm{P}<0.05$ vs. $12 \mu \mathrm{M}$ $\mathrm{MH}$ alone.

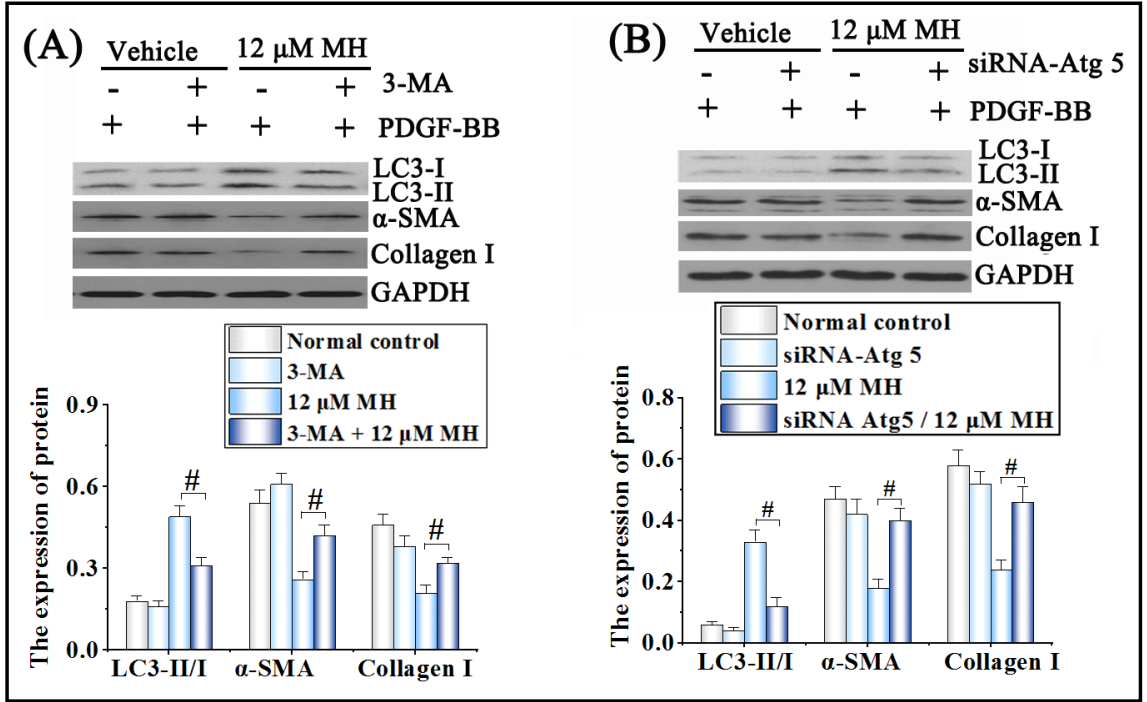

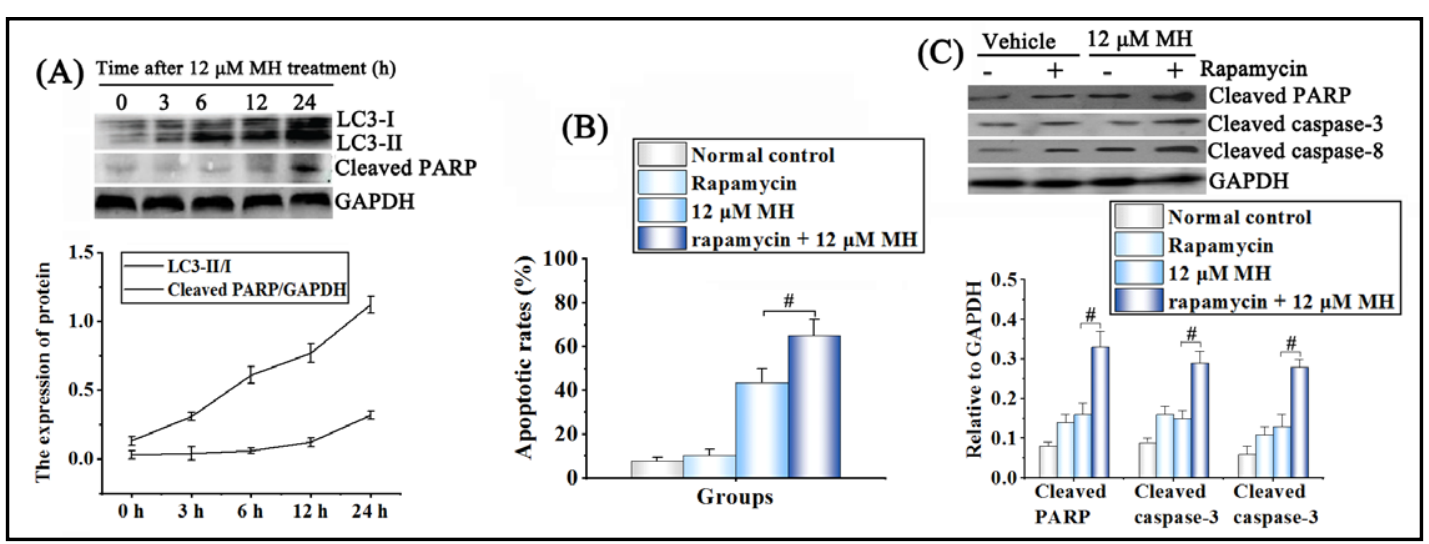

Fig. 6. Induction of autophagy enhanced MH-induced apoptosis in HSCs. (A) Autophagy precedes apoptosis in MH-treated HSCs. (B) - (C) HSC-T6 cells were pretreated with rapamycin for $12 \mathrm{~h}$ and then incubated with $\mathrm{MH}$ for $24 \mathrm{~h}$, and then apoptotic rates and the expression of the apoptosis-relative proteins were detected, respectively. ${ }^{\#} \mathrm{P}<0.05$ vs. $12 \mu \mathrm{M}$ MH alone. 
Inhibition of autophagy impaired the ability of MH in inhibiting HSC activation

HSC-T6 cells were pretreated with PDGF-BB $(10 \mathrm{nmol} / \mathrm{l})$ for $24 \mathrm{~h}$ to induce cell activation followed by 3-Methyl adenine (3-MA) for $12 \mathrm{~h}$, and then wells were treated with $12 \mu \mathrm{M} \mathrm{MH}$ for $24 \mathrm{~h}$. As shown in Fig. 5A, treatment with MH markedly increased the conversion of LC3-I to LC3-II compared with vehicle-treated HSCs, but autophagy specific inhibitor 3-MA treatment largely abolished the promoting effect of $\mathrm{MH}$ on autophagy. Moreover, treatment with $\mathrm{MH}$ markedly decreased the expressions of $\alpha$-SMA and collagen I, whereas 3-MA significantly impaired the ability of MH in inhibiting the expressions of both the proteins. Similarly, Atg5 knockdown (blocking autophagy) also abolished the inhibitory effect of MH on HSC activation (Fig. 5B). Overall, these data demonstrated that inhibition of autophagy impaired the effect of $\mathrm{MH}$ on HSC activation.

\section{Autophagy promoted MH-induced apoptosis}

LC3-II is an autophagy-specific marker. Expression of cleaved PARP occurs downstream of caspase activation, which is a specific marker of apoptosis. In the present study, the expression of both LC3-II and cleaved PARP was significantly increased after MH treatment. However, their changes were obviously not synchronized: the expression of cleaved PARP was significantly increased at $24 \mathrm{~h}$, whereas LC3-II increased markedly at $6 \mathrm{~h}$ (Fig. 6A). These results suggested that autophagy precedes apoptosis in MH-treated HSCs.

As shown in Fig. 6B, treatment with $\mathrm{MH}(12 \mu \mathrm{M})$ increased the apoptotic rate compared with the normal control. Interestingly, application of rapamycin (a specific autophagic promotor, $10 \mathrm{nmol} / \mathrm{l}$ ) could further promoted $\mathrm{MH}$-induced apoptotic rate. Moreover, rapamycin also further increased the levels of the apoptosis-related markers, such as cleaved caspase- 3 and- 8 as well as cleaved PARP in response to MH (Fig. 6C). These results suggested that induction of autophagy promotes $\mathrm{MH}$-induced apoptosis.

\section{JNK signaling pathway involved in MH-induced autophagy and apoptosis}

Autophagy can be induced by activated c-Jun during endoplasmic reticulum (ER) stress response [18]. In the present study, treatment with $\mathrm{MH}$ for $24 \mathrm{~h}$ significantly increased the phosphorylation of JNK and c-Jun (Fig. 7A). Moreover, compared to MH alone, pretreatment with the JNK-specific inhibitor SP600125 for $12 \mathrm{~h}$ not only effectively decreased the expression of Beclin-1 and LC3-II (Fig. 7B), but also decreased the expression of the apoptosis-related

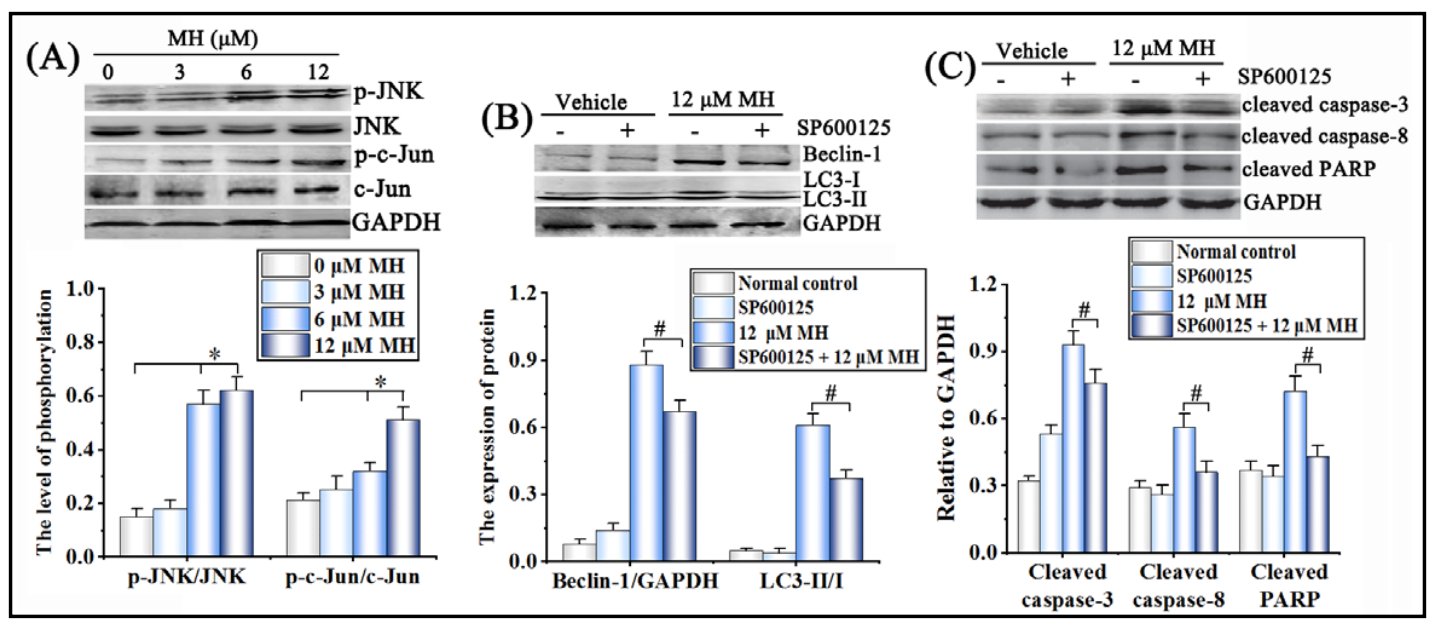

Fig. 7. MH induced autophagy and apoptosis partially by activating the JNK signaling pathway. (A) The phosphorylation of JNK and c-Jun was detected by Western blotting. ${ }^{*} \mathrm{P}<0.05$ vs. the untreated control $(0 \mu \mathrm{M}$ $\mathrm{MH})$. (B-C) The autophagy-relative and apoptosis-relative markers were determined by Western blotting. ${ }^{\#} \mathrm{P}<0.05$ vs. $12 \mu \mathrm{M}$ MH alone. 
Fig. 8. $M H$ induced apoptosis and autophagy by inhibiting the PI3K/ Akt/mTOR pathway in HSC-T6 cells. (A) The phosphorylation of PI3K, mTOR and p70S6k was detected by Western blotting. $\quad{ }^{*} \mathrm{P}<0.05$ vs. the untreated control (0 $\mu \mathrm{M} \mathrm{MH})$. (B) Apoptotic rare was detected by flow cytometry. (C) The autophagy-relative marker LC3-I/II was determined by Western blotting. ${ }^{\#} \mathrm{P}<0.05$ vs. $12 \mu \mathrm{M}$ $\mathrm{MH}$ alone.

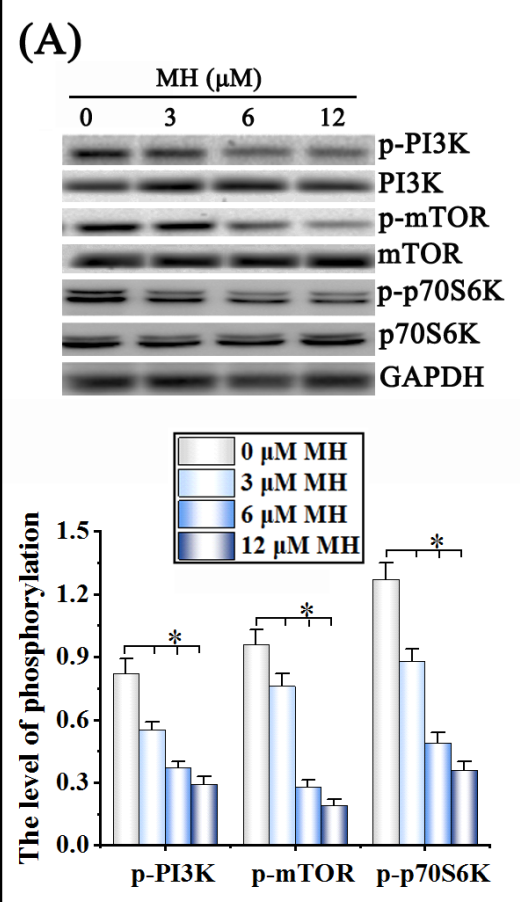

proteins, including cleaved caspase-3, cleaved caspase-8 and cleaved PARP (Fig. 7C). These results suggested that MH induces autophagy and apoptosis in HSCs partially through the activation of the JNK pathway.

MH induced apoptosis and autophagy through inhibition of the PI3K/Akt/mTOR pathway

$\mathrm{PI} 3 \mathrm{~K} / \mathrm{Akt} / \mathrm{mTOR}$ signaling pathway plays an important role in apoptotic and autophagic cell death. In the present study, treatment with $\mathrm{MH}$ for $24 \mathrm{~h}$ decreased the phosphorylation of PI3K, AKT, mTOR and p70S6K in a concentration-dependent manner (Fig. 8A), suggesting that MH significantly inhibits the Akt/mTOR/p70S6K signaling pathway. Moreover, treatment with the PI3K/Akt inhibitor LY294002 alone for $12 \mathrm{~h}$ did not increase the basal apoptosis level, but it did increase the amount of MH-induced apoptosis (Fig. 8B). Similarly, LY294002 alone did not increase the processing of LC3-II, but it did promote MH-induced processing of LC3-I to LC3-II (Fig. 8C). These results indicated that MH induces autophagy and apoptosis also through the inhibition of the PI3K/Akt/mTOR signaling pathway.

\section{Discussion}

HSCs trans-differentiation into myofibroblasts has been identified as an important event in liver fibrogenesis, and therefore inhibiting HSC activation is a key in reversing hepatic fibrosis [2]. In the present study, MH treatment significantly inhibited HSC-T6 cell viability and colony formation in a concentration-dependent manner. MH notably reduced the expressions of $\alpha$-SMA and collagen I. Moreover, MH treatment arrested HSC-T6 cells in G2/M phase. These data suggested that MH significantly inhibits HSC activation.

Previous studies have showed that selective removal of activated HSCs through induction of apoptosis is a therapeutic strategy in hepatic fibrosis [19]. It has been demonstrated that apoptosis is closely related to the mitochondria-dependent pathway. Mitochondrial dysfunction causes the collapse of mitochondria membrane potential (MMP), which results in mitochondrial permeability transition pore (MPTP) opening, enabling to the release of 


\section{Cellular Physiology Cell Physiol Biochem 2018;51:897-908 \begin{tabular}{ll|l} 
and Biochemistry & $\begin{array}{l}\text { DOl: 10.1159/000495390 } \\
\text { Published online: 22 November } 2018\end{array}$ & $\begin{array}{l}\text { O } 2018 \text { The Author(s). Published by S. Karger AG, Basel } \\
\text { ww.karger.com/cpb }\end{array}$ \\
\cline { 2 - 4 }
\end{tabular} \\ Zhang et al.: Methyl Helicterate Inhibits HSC Activation}

cytochrome c from mitochondria into cytoplasm [20]. The mitochondria-dependent apoptotic pathway is regulated by Bcl-2 family of proteins, including Bax and Bcl-2. An increase in the Bax/Bcl-2 ratio has been confirmed to promote apoptosis by directly activating the mitochondrial apoptotic pathway [21]. In addition, apoptosis is caused by proteases, known as "caspases", which specifically target cysteine aspartyl [22]. With respect to the inhibitory effects of MH on HSC activation and collagen synthesis, we observed that treatment with MH significantly induced apoptosis in HSC-T6 cells. As an attempt to elucidate the molecular mechanism involved in $\mathrm{MH}$-induced apoptosis, we observed that $\mathrm{MH}$ significantly decreased the anti-apoptotic proteins Bcl-2 expression, increased the pro-apoptotic proteins Bax level, and caused a loss of MMP and an obvious translocation of cytochrome c from mitochondria to cytosol. Moreover, MH markedly increased the activities of caspase- 3 and -8 . These data indicated that MH-induced HSC apoptosis is mediated by the mitochondria-dependent pathway and the activation of caspases.

Autophagy as a defensive mechanism against liver fibrosis has been identified by accumulating evidences. Chen et al. [23] reported that the ability of Oroxylin A in ameliorating liver fibrosis and inhibiting HSC activation is associated with activation of autophagy. Yang et al. [24] found that the protective effects of caffeic acid phenethyl ester against liver fibrosis might due to the inhibition of TGF- $\beta 1 /$ Smad3 signaling and induction of authophagy in HSCs. Moreover, Zhang et al. [25] also demonstrated that dihydroartemisinin suppressed inflammation in liver fibrosis model by inducing autophagy pathway. In the present study, MH treatment significantly increased lysosomes and autophagosomes in a concentrationdependent manner in HSC-T6 cells. MH notably increased the formation of autophagic vacuoles and enhanced autophagic flux. Furthermore, MH markedly increased the conversion of LC3-I to LC3-II, upregulated the expressions of Beclin-1, Atg 5 and 7, but reduced the expression of P62. Interestingly, inhibition of autophagy by 3-MA or Atg5 knockdown abolished the ability of MH in inhibiting HSC activation. In contrast, induction of autophagy by rapamycin promoted MH-induced HSC apoptosis. Our results suggested that autophagy induced by MH may be an important underlying mechanism for the anti-fibrosis effect of $\mathrm{MH}$, to some extent.

The JNK pathway is well known to be involved in hepatic fibrogenesis and HSC activation [26]. In this study, MH treatment significantly induced the activation of the JNK pathway. Interestingly, pretreatment with the JNK-specific inhibitor SP600125 not only effectively blocked MH-induced autophagy, but also decreased the expression of the apoptosis-related proteins, suggesting that $\mathrm{MH}$ induces autophagy and apoptosis in HSCs partially through activating the JNK pathway. Moreover, the Akt/mTOR/p70S6k signaling pathway is also involved in liver fibrogenesis and HSC activation, as the use of a number of inhibitors of this pathway alleviated liver fibrosis and decreased HSC activation [27]. In the present study, treatment with MH for $24 \mathrm{~h}$ decreased the phosphorylation of PI3K, AKT, mTOR and p70S6K in a concentration-dependent manner, suggesting an inhibitory effect of $\mathrm{MH}$ on the Akt/ mTOR/p70S6K pathway. Importantly, treatment with the PI3K/Akt inhibitor LY294002 did increase the amount of $\mathrm{MH}$-induced apoptosis and promote the $\mathrm{MH}$-induced processing of LC3-II, suggesting that MH induces autophagy and apoptosis also through the inhibition of the PI3K/Akt/mTOR pathway.

\section{Conclusion}

The present study demonstrates that MH significantly inhibits HSC-T6 cell activation and induces cell cycle arrest at G2/M phase. Moreover, it markedly induces HSC apoptosis and autophagy via regulating the JNK and PI3K/Akt/mTOR pathways. The present study provides novel insights into the anti-fibrotic mechanism of $\mathrm{MH}$ and highlights the complex interplay between apoptosis and autophagy. 


\section{Cellular Physiology Cell Physiol Biochem 2018;51:897-908 \begin{tabular}{ll|l} 
and Biochemistry & $\begin{array}{l}\text { DOI: } 10.1159 / 000495390 \\
\text { Published onlIne: } 22 \text { November } 2018\end{array}$ & $\begin{array}{l}\text { (c) } 2018 \text { The Author(s). Published by S. Karger AG, Basel } \\
\text { www.karger.com/cpb }\end{array}$
\end{tabular} \\ Zhang et al.: Methyl Helicterate Inhibits HSC Activation}

\section{Acknowledgements}

The authors gratefully acknowledge the financial support provided by the National Natural Science Foundation of China (No.81473431; No.81660693; No.81660686; No.81660706) and the Guangxi Natural Science Foundation (2016GXNSFDA380025).

\section{Disclosure Statement}

The authors declare that there are no conflicts of interest.

\section{References}

1 Wallace K, Burt A, Wright M: Liver fibrosis. Biochem J 2008;411:1-18.

-2 Gülsüm, zlem, Elpek: Cellular and molecular mechanisms in the pathogenesis of liver fibrosis: An update. World J Gastroenterol 2014;20:7260-7276.

$>3$ Thoen LFR, Guimarã£Es EL, Grunsven LAV: Autophagy: A new player in hepatic stellate cell activation. Autophagy 2012;8:126-128.

- Wang B, Yang H, Fan Y, Yang Y, Cao W, Jia Y, Cao Y, Sun K, Pang Z, Du H: 3-methyladenine ameliorates liver fibrosis through autophagy regulated by the NF- $\kappa \mathrm{B}$ signaling pathways on hepatic stellate cell. Oncotarget 2017;8:107603-107611.

5 Ni HM, Williams JA, Yang H, Shi YH, Fan J, Ding WX: Targeting autophagy for the treatment of liver diseases. Pharmacol Res 2012;66:463-474.

6 Boya P, Codogno P: Micronucleophagy: A new mechanism to protect against chromosomal instability? Cell Cycle 2012;11:645.

-7 Lin X, Huang R, Zhang S, Zheng L, Wei L, He M, Zhou Y, Zhuo L, Huang Q: Methyl helicterate protects against $\mathrm{CCl}_{4}$-induced liver injury in rats by inhibiting oxidative stress, NF- $\kappa$ B activation, Fas/Fasl pathway and cytochrome p4502e1 level. Food Chem Toxicol 2012;50:3413-3420.

-8 Huang Q Huang R, Wei L, Chen Y, Lv S, Liang C, Zhang X, Yin F, Li H, Zhuo L: Antiviral activity of methyl helicterate isolated from helicteres angustifolia (sterculiaceae) against hepatitis B virus. Antiviral Res 2013;100:373-381.

-9 Huang Q Li Y, Zhang S, Huang R, Zheng L, Wei L, He M, Liao M, Li L, Zhuo L: Effect and mechanism of methyl helicterate isolated from helicteres angustifolia (sterculiaceae) on hepatic fibrosis induced by carbon tetrachloride in rats. J Ethnopharmacol 2012;143:889-895.

10 Bai F, Huang Q, Nie J, Lu S, Lu C, Zhu X, Wang Y, Zhuo L, Lu Z, Lin X: Trolline ameliorates liver fibrosis by inhibiting the NF- $\kappa B$ pathway, promoting hsc apoptosis and suppressing autophagy. Cell Physiol Biochem 2017;44:436-446.

11 Bai F, Huang Q, Wei J, Lv S, Chen Y, Liang C, Wei L, Lu Z, Lin X: Gypsophila elegans isoorientin-2"-o- $\alpha$-larabinopyranosyl ameliorates porcine serum-induced immune liver fibrosis by inhibiting nf-kappab signaling pathway and suppressing hsc activation. Int Immunopharmacol 2018;54:60-67.

12 Lin X, Bai F, Nie J, Lu S, Lu C, Zhu X, Wei J, Lu Z, Huang Q: Didymin alleviates hepatic fibrosis through inhibiting erk and PI3K/Akt pathways via regulation of raf kinase inhibitor protein. Cell Physiol Biochem 2016;40:1422-1432.

$\checkmark 13$ Cossarizza A, Baccaranicontri M, Kalashnikova G, Franceschi C: A new method for the cytofluorometric analysis of mitochondrial membrane potential using the j-aggregate forming lipophilic cation $5,5^{\prime}, 6$, $6^{\prime}$-tetrachloro-1, 1',3, 3'-tetraethylbenzimidazolcarbocyanine iodide (JC-1). Biochem Biophys Res Commun 1993;197:40-45.

14 Zamzami N, Marchetti P, Castedo M, Decaudin D, Macho A, Hirsch T, Susin SA, Petit PX, Mignotte B, Kroemer G: Sequential reduction of mitochondrial transmembrane potential and generation of reactive oxygen species in early programmed cell death. J Exp Med 1995;182:367-377.

15 Lin X, Wei J, Chen Y, He P, Lin J, Tan S, Nie J, Lu S, He M, Lu Z, Huang Q: Isoorientin from gypsophila elegans induces apoptosis in liver cancer cells via mitochondrial-mediated pathway. J Ethnopharmacol 2016;187:187-194. 


\section{Cellular Physiology Cell Physiol Biochem 2018;51:897-908 \begin{tabular}{ll|l} 
and Biochemistry & DOl: 10.1159/000495390 & $\begin{array}{l}\text { O } 2018 \text { The Author(s). Published by S. Karger AG, Basel } \\
\text { www.karger.com/cpb }\end{array}$ \\
\cline { 1 - 3 }
\end{tabular}}

Zhang et al.: Methyl Helicterate Inhibits HSC Activation

16 Huang Q Liang C, Wei L, Nie J, Lu S, Lu C, Zhuo L, Lu Z, Lin X: Raf kinase inhibitory protein down-expression exacerbates hepatic fibrosis in vivo and in vitro. Cell Physiol Biochem 2016;40:49-61.

17 Mizushima N, Yoshimori T: How to interpret lc3 immunoblotting. Autophagy 2007;3:542-545.

18 Fuest M, Willim K, MacNelly S, Fellner N, Resch GP, Blum HE, Hasselblatt P: The transcription factor c-jun protects against sustained hepatic endoplasmic reticulum stress thereby promoting hepatocyte survival. Hepatology 2012;55:408-418.

19 Galluzzi L, Pedro BS, Vitale I, Aaronson SA, Abrams JM, Adam D, Alnemri ES, Altucci L, Andrews D, Annicchiaricopetruzzelli M: Essential versus accessory aspects of cell death: Recommendations of the nccd 2015. Cell Death Differ 2015;22:58-73.

-20 Wu W, Zhou X, Liu P, Fei W, Li L, Yun H: Isoflurane reduces hypoxia/reoxygenation-induced apoptosis and mitochondrial permeability transition in rat primary cultured cardiocytes. BMC Anesthesiol 2014:14-17.

21 Brunelle JK, Letai A: Control of mitochondrial apoptosis by the bcl-2 family. J Cell Sci 2009;122:437-441.

22 Thornberry NA: Caspases: Key mediators of apoptosis. Chem Biol 1998;5:97-103.

-23 Chen W, Zhang Z, Yao Z, Wang L, Zhang F, Shao J, Chen A, Zheng S: Activation of autophagy is required for oroxylin a to alleviate carbon tetrachloride-induced liver fibrosis and hepatic stellate cell activation. Int Immunopharmacol 2018;56:148-155.

24 Yang N, Dang S, Shi J, Wu F, Li M, Zhang X, Li Y, Jia X, Zhai S: Caffeic acid phenethyl ester attenuates liver fibrosis via inhibition of TGF- $31 /$ smad3 pathway and induction of autophagy pathway. Biochem Biophys Res Commun 2017;486:22-28.

25 Zhang Z, Mei G, Zhao S, Shao J, Zheng S: Ros-jnk1/2-dependent activation of autophagy is required for the induction of anti-inflammatory effect of dihydroartemisinin in liver fibrosis. Free Radic Biol Med 2016;101:272-283.

-26 Parsons CJ, Takashima M, Rippe RA: Molecular mechanisms of hepatic fibrogenesis. J Gastroenterol Hepatol 2010;22:S79-S84.

-27 Li J, Li X, Xu W, Wang S, Hu Z, Zhang Q, Deng X, Wang J, Zhang J, Guo C: Antifibrotic effects of luteolin on hepatic stellate cells and liver fibrosis by targeting Akt/mTOR/p70S6K and TGF $\beta /$ smad signalling pathways. Liver International 2015;35:1222-1233. 